\title{
Upregulated SMAD3 promotes epithelial-mesenchymal transition and predicts poor prognosis in pancreatic ductal adenocarcinoma
}

\author{
Ken Yamazaki ${ }^{1}$, Yohei Masugi ${ }^{1}$, Kathryn Effendi ${ }^{1}$, Hanako Tsujikawa ${ }^{1}$, Nobuyoshi Hiraoka ${ }^{2}$, Minoru Kitago ${ }^{3}$, \\ Masahiro Shinoda ${ }^{3}$, Osamu Itano ${ }^{3}$, Minoru Tanabe ${ }^{3}$, Yuko Kitagawa ${ }^{3}$ and Michiie Sakamoto ${ }^{1}$
}

In pancreatic ductal adenocarcinoma (PDAC), features of epithelial-mesenchymal transition (EMT) are often seen in tumor tissue, and such features correlate with poor prognosis. Solitary infiltration of tumor cells represents a morphological phenotype of EMT, and we previously reported that a high degree of solitary cell infiltration correlates with EMT-like features, including reduced E-cadherin and elevated vimentin levels. Using solitary cell infiltration to evaluate the degree of EMT, gene-expression profiling of 12 PDAC xenografts was performed, and SMAD3 was identified as an EMT-related gene. Immunohistochemistry using clinical specimens $(n=113)$ showed that SMAD3 accumulated in the nuclei of tumor cells, but was not detected in most epithelial cells in the pancreatic duct. Moreover, SMAD3 upregulation correlated with malignant characteristics, such as higher tumor grade and lymph node metastasis, as well as with EMT-like features. SMAD4, which plays a key role in transforming growth factor- $\beta$ (TGF- $\beta$ ) signaling, is inactivated in approximately half of PDAC cases. In this study, the nuclear accumulation of SMAD3 was immunohistochemically detected even in SMAD4-negative cases. SMAD3 knockdown resulted in upregulated E-cadherin, downregulated vimentin, and reduced cell motility in pancreatic cancer cells regardless of SMAD4 status. In addition, TGF- $\beta$-treatment resulted in EMT induction in cells carrying wild-type SMAD4, and EMT was suppressed by SMAD3 knockdown. Patients with upregulated SMAD3 and a high degree of solitary cell infiltration had shorter times to recurrence and shorter survival times after surgery, and multivariate analysis showed that both factors were independent prognostic factors linked to unfavorable outcomes. These findings suggest that SMAD3 in PDAC is involved in the promotion of malignant potential through EMT induction in tumor cells regardless of SMAD4 status and serves as a potential biomarker of poor prognosis.

Laboratory Investigation (2014) 94, 683-691; doi:10.1038/labinvest.2014.53; published online 7 April 2014

KEYWORDS: epithelial-mesenchymal transition; pancreatic ductal adenocarcinoma; prognosis; SMAD3; TGF- $\beta$

Pancreatic ductal adenocarcinoma (PDAC) is a leading cause of cancer death worldwide. ${ }^{1}$ Despite recent advances in diagnosis and treatment, the prognosis of patients with PDAC is still extremely poor. Many pathologic features (eg, margin status, tumor size, tumor grade, and lymph node metastasis) have been shown to correlate with poor prognosis. ${ }^{2-4}$ In addition, PDACs also often display the intratumoral heterogeneity of glandular differentiation. Among heterogeneous tumor cells, those capable of independently infiltrating into the stroma have the most dedifferentiated morphology and are frequently found at the invasion front. Moreover, we previously reported that an increase in the number of solitary infiltrating cells correlates with epithelial-mesenchymal transition (EMT)-like features, such as E-cadherin downregulation and vimentin upregulation. ${ }^{5}$

EMT-like features are often seen in many types of cancer. Tumor cells with epithelial cell characteristics have apicalbasal polarity and are organized in cell layers with cell-cell adhesion, whereas those with mesenchymal characteristics lose this polarity and migrate to the extracellular matrix. During EMT, tumor cells lose epithelial markers, such as E-cadherin and certain cytokeratins, and gain mesenchymal

\footnotetext{
${ }^{1}$ Department of Pathology, School of Medicine, Keio University, Tokyo, Japan; ${ }^{2}$ Pathology Division, National Cancer Center Research Institute, Tokyo, Japan and ${ }^{3}$ Department of Surgery, School of Medicine, Keio University, Tokyo, Japan

Correspondence: Professor M Sakamoto, MD, PhD, Department of Pathology, School of Medicine, Keio University, 35 Shinanomachi, Shunjuku-ku, Tokyo 160-8582, Japan.

E-mail: msakamot@z5.keio.jp
}

Received 29 October 2013; revised 10 February 2014; accepted 13 February 2014 
markers such as vimentin and fibronectin. ${ }^{6,7}$ Similarly, tumor cells in the standard cancer metastasis model dissociate from the tumor cell mass with tight cell-cell adhesion, invade the stroma, intravasate, circulate through the body, and form a secondary focus at a distant site. Thus, EMT can be considered an early event in cancer metastasis.

EMT is induced by extracellular signals including soluble factors, such as those in the transforming growth factor- $\beta$ (TGF- $\beta$ ) super family, fibroblast growth factor families, and epidermal growth factor, as well as components of the extracellular matrix. ${ }^{8}$ Overexpression of TGF- $\beta$ in PDAC correlates with poor prognosis. ${ }^{9}$ Interestingly, the SMAD4 gene, which plays a key role in the TGF- $\beta$ signaling pathway, is inactivated by mutations and deletions in $55 \%$ of PDAC cases. ${ }^{10}$ Although TGF- $\beta$ signaling seems to be abrogated by SMAD4 inactivation, nuclear accumulation of SMAD2 and SMAD3 has been found in SMAD4-null cells, indicating the presence of SMAD4-independent nuclear translocation of SMAD2 and SMAD3. ${ }^{11}$ In PDAC, SMAD4 mutations are associated with poor patient outcome. ${ }^{10,12}$ Moreover, EMTlike features such as E-cadherin downregulation and an increased number of solitary infiltrating cells are also frequently found in PDACs, and such features correlate with poor prognosis. ${ }^{5,13}$ These findings suggest that a SMAD4-independent signaling pathway could induce EMTlike features in PDAC.

Understanding the mechanism responsible for the induction of EMT in PDAC may contribute to improved diagnosis and treatment of patients. Previously, we showed that solitary cell infiltration serves as a morphological clue to EMT in PDAC. ${ }^{5}$ In the current work, based on solitary cell infiltration as an indicator of EMT, we reanalyze gene expression profile of PDAC xenografts ${ }^{14}$ in order to search for EMT-related genes and examine their role in PDAC.

\section{MATERIALS AND METHODS Clinical Specimens}

PDAC tissues were obtained from patients who underwent surgical resection between 1995 and 2004 at Keio University Hospital $(n=113)$ and the National Cancer Center Hospital, Japan $(n=12) .{ }^{14}$ All experiments using human samples were approved by the ethics committees of Keio University and the National Cancer Center. Tumor grade was evaluated according to the WHO tumor grading system. ${ }^{15}$

\section{Microarray Analysis}

Previously reported microarray data of 12 PDAC xenografts ${ }^{14}$ were reanalyzed. Minimum information about a microarray experiment (MIAME)-compliant microarray data (experiment ID EXPR053) are accessible from our database, Genome Medicine Database of Japan (GeMDBJ; https:// gemdbj.nibio.go.jp/dgdb/DownloadSite.do). Expression profiles were clustered using established algorithms implemented in the software program Cluster 2.0. Centroid linkage clustering with uncentered correlation was used, and TreeView software
(http://rana.lbl.gov/EisenSoftware.htm) generated a visual representation of clusters.

\section{Immunohistochemistry}

Immunohistochemical staining using PDAC tissues from 113 patients was performed according to the method as previously described. ${ }^{5}$ Rabbit polyclonal anti-SMAD3 antibody was obtained from Invitrogen (Carlsbad, CA, USA) and used in a 1:200 dilution. Sections were counterstained with hematoxylin. The medical records of all consecutive patients who underwent resection with curative intent for PDAC at Keio University Hospital were reviewed to examine the correlation with SMAD3 immunostaining.

\section{In Vitro Analyses}

Human PDAC cell lines AsPC-1, CFPAC-1, and PANC-1 were obtained from the American Type Culture Collection (Manassas, VA, USA). RNA interference (RNAi) was performed as previously described ${ }^{14}$ using two siRNA molecules targeting the following sequences in SMAD3 mRNA: siSMAD3a, 5'-CC AGUGACCACCAGAUGAA- $3^{\prime}$ and siSMAD $3 b, 5^{\prime}$-GGAGAAA UGGUGCGAGAAG- $3^{\prime}$. These siRNAs and negative control siRNA were obtained from QIAGEN (Valencia, CA, USA). At 2 or 3 days after siRNA transfection, the transfectants were harvested for western blot and real-time RT-PCR analyses as previously described. ${ }^{14,16}$ All primer sequences are shown in Supplementary Table 1.

At $24 \mathrm{~h}$ after transfection, cells were incubated with TGF- $\beta$ ( $5 \mathrm{ng} / \mathrm{ml}$; Sigma-Aldrich, St Louis, MO, USA) for $24 \mathrm{~h}$ and then harvested. Migration assays using Transwell inserts (Costar, Cambridge, MA, USA) were performed as previously described. $^{14}$ The number of migrated cells/field was determined by counting three fields from each transwell. Assays were performed in triplicate. Percent migration was defined as the ratio of the mean number of migrated SMAD3-knockdown cells to the mean number of migrated cells treated with negative control siRNA.

\section{Statistical Analyses}

Statistical analyses were performed using the Statistical Package for the Social Sciences (SPSS, Chicago, IL, USA), version 19.0 , and $P$-values of $<0.05$ were considered statistically significant. Survival curves were calculated from the date of surgery using the Kaplan-Meier method and were compared using the log-rank test. Multivariate analyses were examined using the Cox proportional hazards regression model.

\section{RESULTS}

\section{EMT Signature in PDACs}

To identify EMT-related genes in PDAC, we reanalyzed gene expression profiles of xenografts derived from 12 PDAC patients ${ }^{14}$ on the basis of the degree of solitary cell infiltration as an EMT-like feature. Previously, we showed that a high degree of solitary cell infiltration $\left(\mathrm{SCI}^{\text {high }}\right.$; seven or more solitary infiltrating tumor cells per 10 fields) significantly 
correlates with EMT-like features including E-cadherin downregulation and vimentin upregulation. ${ }^{5}$ Eight samples showed $\mathrm{SCI}^{\text {high }}$ and were classified as the high-EMT group (H1-H8), whereas four samples were classified as the lowEMT group (L1-L4) (Supplementary Table 2). Genes differentially expressed between the two groups were extracted based on the following criteria: mean signal (expression level) in one of the two groups is $\geq 1000$ (when mean signal of all probe sets was normalized to 1000 in each microarray) and $\geq 2$-fold that of the other group, with a $P$-value (Student's $t$ test) of $\leq 0.05$. Figure 1a shows the result of two-way clustering analysis using 53 genes (66 probe sets) extracted as described above. The degree of solitary cell infiltration has been shown to correlate with vimentin upregulation and E-cadherin downregulation on immunohistochemical analyses; ${ }^{5}$ however, these two genes did not satisfy the above differential expression criteria. This finding indicates that tumor cells showing such features were localized to the focal lesions, and their expression levels were not reflected in the tumor tissue as a whole.

One of the upregulated genes in the high-EMT group that met the differential expression criteria was the SMAD3 gene. SMAD3 acts as a mediator of TGF- $\beta$ signaling that is known to be involved in the EMT process. To confirm the differential SMAD3 expression levels between the two groups, immunohistochemistry was performed using the same tumor tissues that had been used for microarray analyses $(\mathrm{H} 2, \mathrm{H} 4$, L2, and L3 in Figure 1b). Increased positivity of SMAD3 in tumor cells was found in the high-EMT group compared with the low-EMT group. These results suggest that enhanced SMAD3 expression at the mRNA and protein levels may be related to EMT-like features in PDAC.

\section{Upregulation of SMAD3 Correlates with PDAC Malignancy}

To examine SMAD3 expression in clinical specimens, immunohistochemical analyses were performed using 113 cases of PDAC. Among these cases, the percentage of tumor cells with SMAD3-positive nuclear staining was highly heterogeneous (mean, 17.6\%; median, 10\%; range, 0-75\%). SMAD3 was detected within the nucleus in almost all SMAD3-positive tumor cells, whereas nuclear accumulation of SMAD3 in epithelial cells of the pancreatic duct was rarely seen (Figure 2 and Supplementary Figure 1). These 113 cases were classified into two groups based on SMAD3 immunopositivity in the tumor cells (SMAD $3^{\text {high }}, \geq 15 \%$ positivity; SMAD3 $3^{\text {low }},<15 \%$ ), and SMAD3 status was compared with clinicopathological parameters (Table 1). SMAD $3^{\text {high }}$ correlated with larger tumor size, major vessel involvement, higher tumor grade, and lymph node metastasis. Furthermore, SMAD $3^{\text {high }}$ also correlated with reduced E-cadherin and elevated vimentin levels as well as a high degree of solitary cell infiltration (Table 1). SMAD4 was detected in $41 \%$ of PDAC cases; however, no correlation was observed between the immunostaining status of SMAD3 and
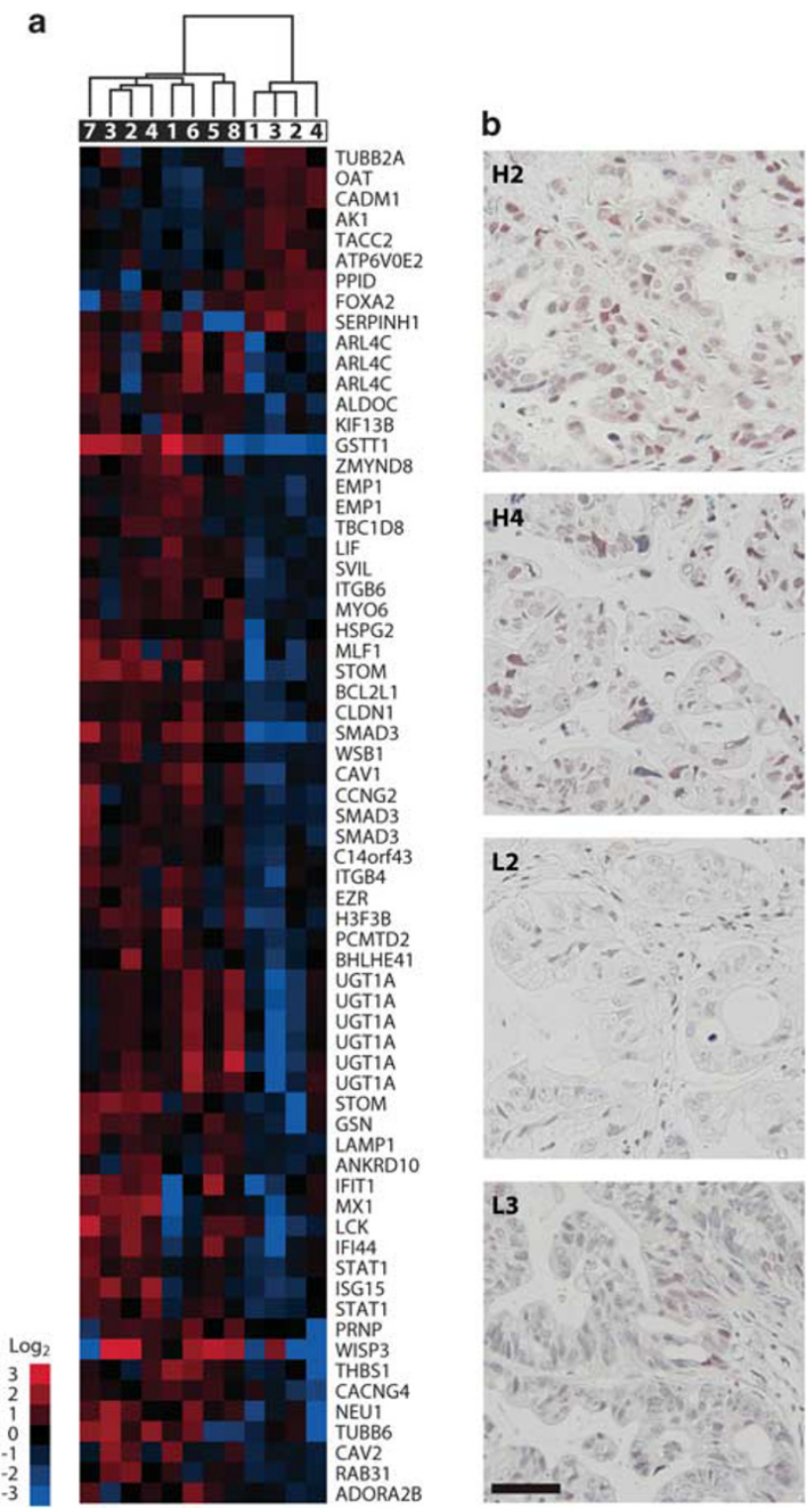

Figure 1 Two-dimensional clustering analysis of the genes differentially expressed between high- and low-EMT groups. (a) The vertical and horizontal axes of the heat map represent genes and tumor samples, respectively. White numbers indicate sample numbers of the high-EMT group ( $\mathrm{H} 1-\mathrm{H} 8)$, and black numbers indicate the low-EMT group (L1-L4). Gene expression levels are represented by the $\log _{2}$ mean intensity ratio and depicted in terms of color variation from red (high expression) to blue (low expression). (b) Expression of SMAD3 was confirmed by immunohistochemistry using tumor tissues ( $\mathrm{H} 2, \mathrm{H} 4, \mathrm{~L} 2$, and L3). Scale bar $=100 \mu \mathrm{m}$.

SMAD4 $(P=0.146)$. In SMAD4-negative cases, nuclear accumulation of SMAD3 with reduced E-cadherin expression was frequently observed at the invasion front of tumor cells (Figure 2 and Supplementary Figure 1).

\section{SMAD3 Knockdown Reduces EMT-Like Features}

SMAD3 expression levels were examined in representative PDAC cell lines. Compared with other gastrointestinal cancer 


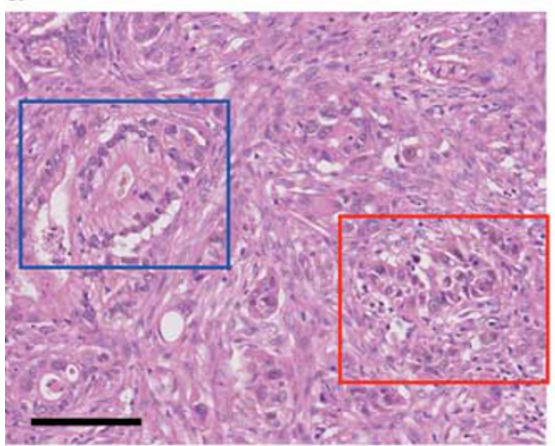

d

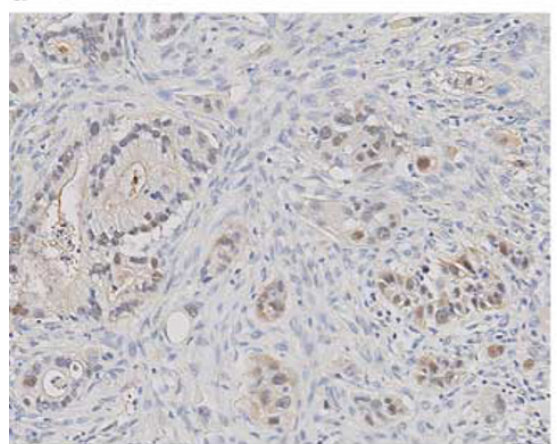

g

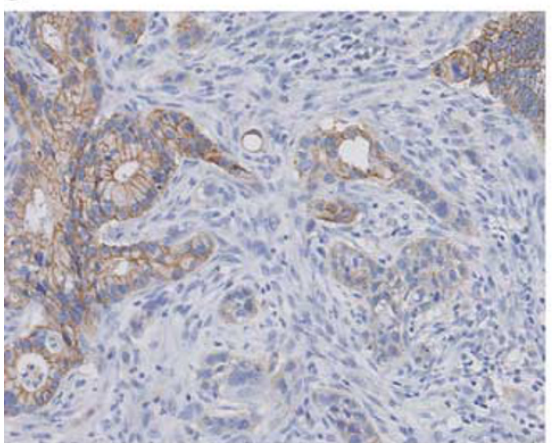

j

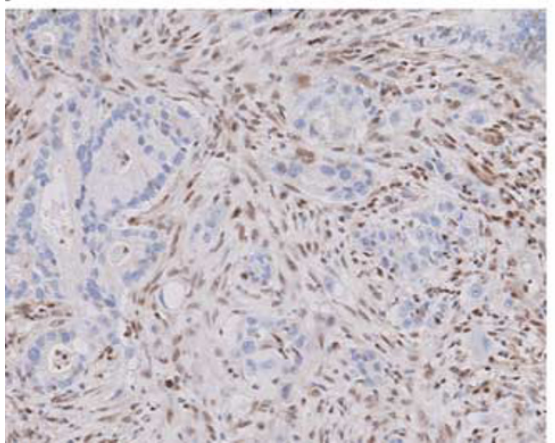

b

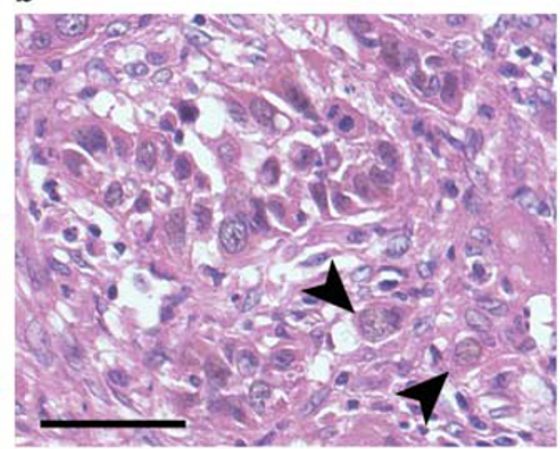

e

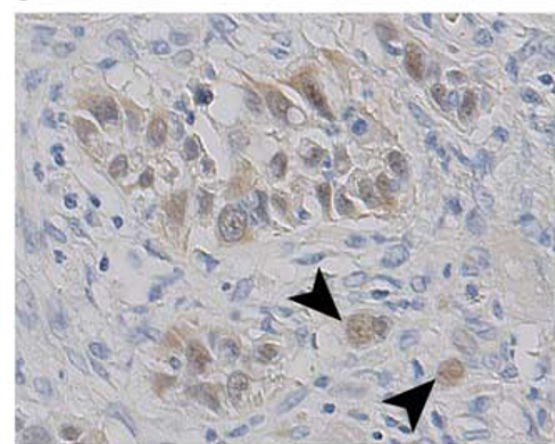

h

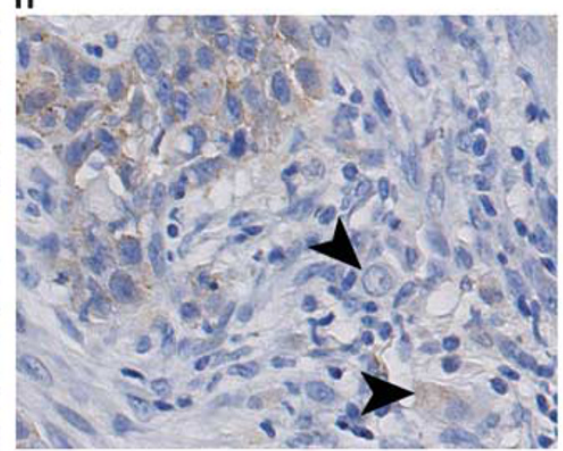

C

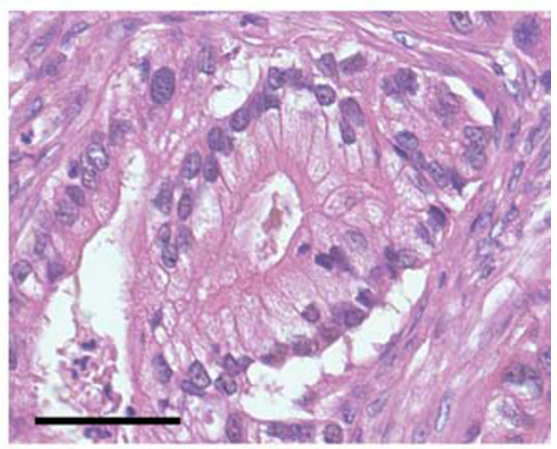

f

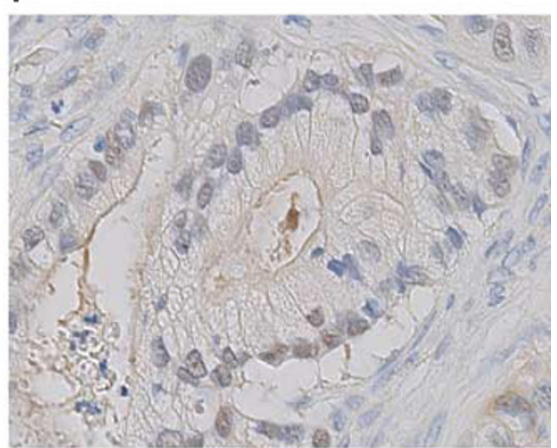

i
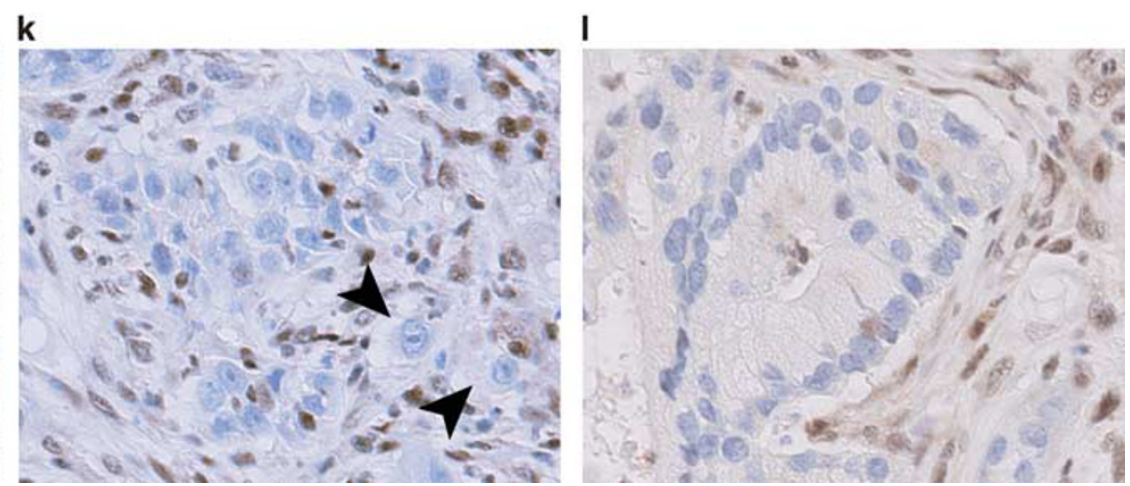

Figure 2 Immunohistochemical detection of SMAD3, E-cadherin, and SMAD4. Serial sections derived from formalin-fixed, paraffin-embedded pancreatic cancer tissue were stained with H\&E (a-c) or DAB followed by probing with anti-SMAD3 (d-f), anti-E-cadherin ( $\mathbf{g}-\mathbf{i})$, and anti-SMAD4 (j-l) antibodies. Solitary infiltrating cells were frequently observed (arrowheads) at the invasive front (red box in $\mathbf{a} ; \mathbf{b}, \mathbf{e}, \mathbf{h}, \mathbf{k}$, high-power fields) compared with the tumor center (blue box in $\mathbf{a} ; \mathbf{c}, \mathbf{f}, \mathbf{i}, \mathbf{l}$, high-power fields). Lymphocytes and stromal cells showed SMAD4-positive nuclei. Scale bars represent $100 \mu \mathrm{m}$ (a) and $50 \mu \mathrm{m}(\mathbf{b}, \mathbf{c})$. 
Table 1 Correlation between clinicopathological characteristics and SMAD3 immunolabeling

\begin{tabular}{|c|c|c|c|}
\hline \multirow[b]{2}{*}{ Characteristics } & \multicolumn{2}{|c|}{ SMAD3-positive rate } & \multirow{2}{*}{$\frac{\chi^{2} \text { test }}{P \text {-value }}$} \\
\hline & $<15 \%$ & $\geq 15 \%$ & \\
\hline Mean age (years old) & 64.8 & 65.3 & $0.786^{\mathrm{a}}$ \\
\hline Gender & & & 0.901 \\
\hline Female & 21 & 20 & \\
\hline Male & 36 & 36 & \\
\hline
\end{tabular}

Mean tumor size $(\mathrm{cm})$

$0.005^{\mathrm{a}}$

Tumor location

Pancreatic head

37

Pancreatic body/tail

Large vessel involvement

Negative

Positive

Neural invasion

Negative

Positive

52

Lymph node metastasis

Negative

Positive

Margin status

Negative

Positive

Tumor grade
$1-2$
3

SMAD4 status

Negative

Positive
1

55

0.457

0.025

$0.212^{b}$

$<0.001$

0.788

40

16

$<0.001$

0.146

0.028

0.002
Table 1 (Continued)

\begin{tabular}{lcccc}
\hline & \multicolumn{2}{c}{ SMAD3-positive rate } & & $\chi^{2}$ test \\
\cline { 2 - 2 } Characteristics & $<15 \%$ & $\geq 15 \%$ & & $P$-value \\
\hline Degree of solitary cell infiltration & & & $<0.001$ \\
Low & 26 & 8 & \\
High & 31 & 48 & \\
\hline
\end{tabular}

aStudent's $t$-test.

${ }^{\mathrm{b}}$ Fisher's exact test.

cell lines, PDAC cell lines commonly showed higher SMAD3 mRNA levels (Supplementary Figure 2). The AsPC-1, CFPAC-1, and PANC-1 cell lines were then selected for further in vitro analyses. Although SMAD4 is inactivated in AsPC-1 and CFPAC-1 cells, ${ }^{17,18}$ SMAD3 expression was detected in the nuclei of these cells, as well as in PANC-1 cells that carry the wild-type SMAD4 allele (Supplementary Figure 3). TGF- $\beta$ induced morphological change and growth arrest in PANC-1 cells, whereas no responses were observed in SMAD4-inactivated cells (Supplementary Figures 4 and 5).

Treatment of these three cell lines with siRNA molecules targeting SMAD3 resulted in decreased SMAD3 expression at both the mRNA and protein levels (Figure 3 and Supplementary Figure 6). In PANC-1 cells, TGF- $\beta$ treatment induced EMT features as shown by E-cadherin downregulation and vimentin upregulation (compare control + TGF- $\beta$ with control - TGF- $\beta$ in Figure $3 b$ ); however, SMAD3 knockdown suppressed these EMT features (compare siSMAD3 + TGF- $\beta$ with control + TGF- $\beta$ ). SMAD3 knockdown also suppressed TGF- $\beta$-induced morphological change and CDKN1A (p21) upregulation in PANC-1 cells (Supplementary Figure 6). These results suggest that TGF- $\beta$-induced EMT is a SMAD3- and SMAD4-dependent process. In SMAD4inactivated AsPC-1 and CFPAC-1 cells, SMAD3 knockdown resulted in increased E-cadherin and reduced vimentin gene expression (compare siSMAD3 - TGF- $\beta$ with control - TGF- $\beta$ ), suggesting that SMAD3 is involved in SMAD4-independent EMT and that TGF- $\beta$ is not required for SMAD4-independent, SMAD3-induced EMT at least in these two cell lines. After SMAD3 knockdown, AsPC-1 cells formed larger cell clusters with decreased cell scattering, whereas CFPAC-1 and PANC-1 cells showed reduced lamellipodial protrusions compared with controls (Figure 3c). To examine whether these morphological changes could contribute to reduced cell motility, migration assays were performed. SMAD3 knockdown decreased the number of cells that migrated through Transwell inserts in all cell lines (Figure 3d).

\section{SMAD3 ${ }^{\text {high }}$ Correlates with Poor Prognosis}

Univariate analysis showed that, in addition to SMAD $3^{\text {high }}$, EMT-like features such as reduced E-cadherin, elevated vimentin, and $\mathrm{SCI}^{\text {high }}$ correlated with shorter survival times 
a

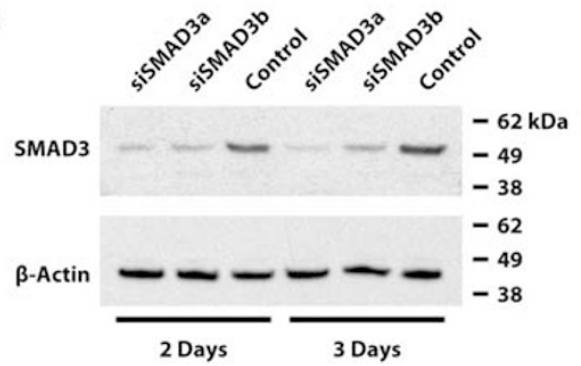

b
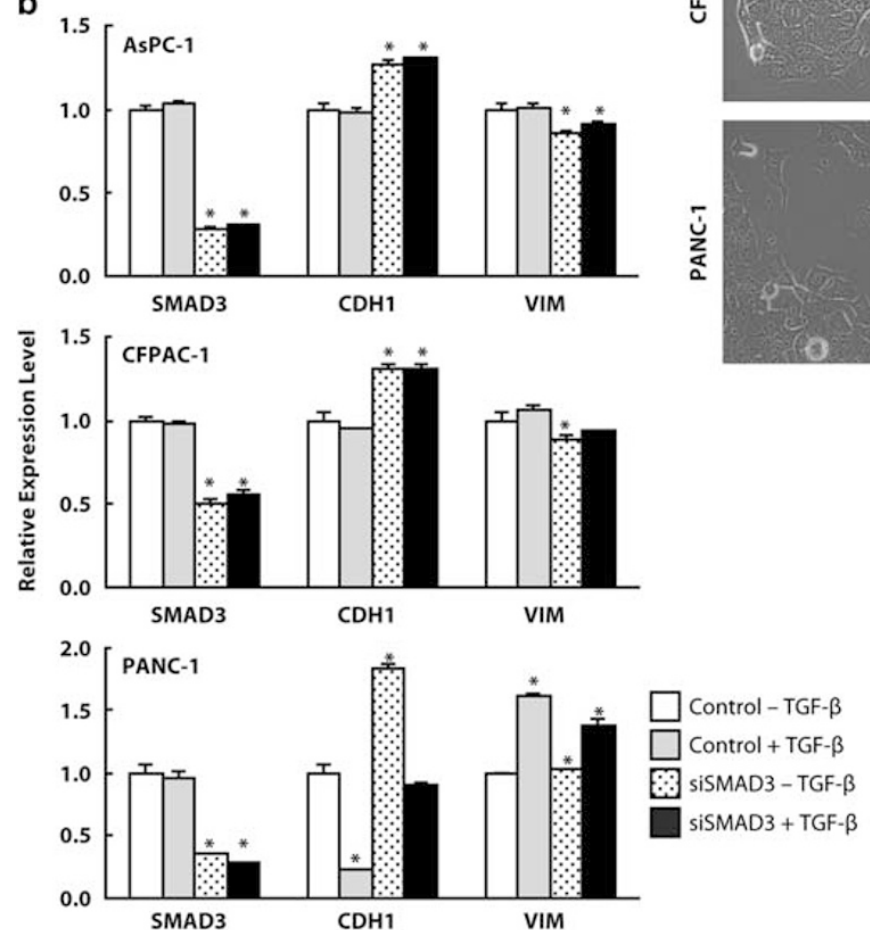

c
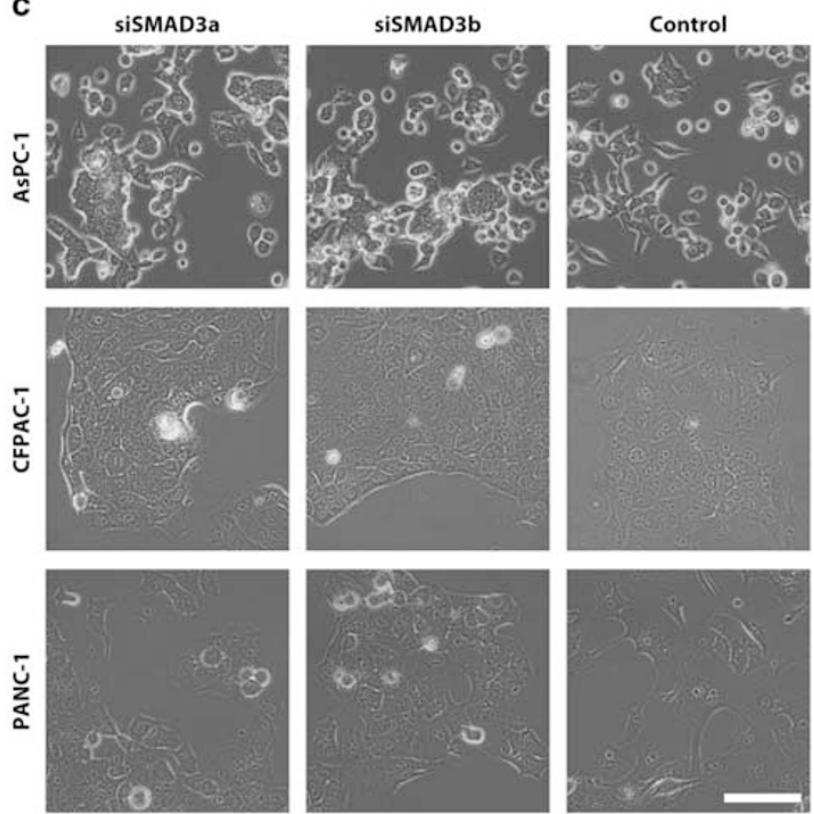

d

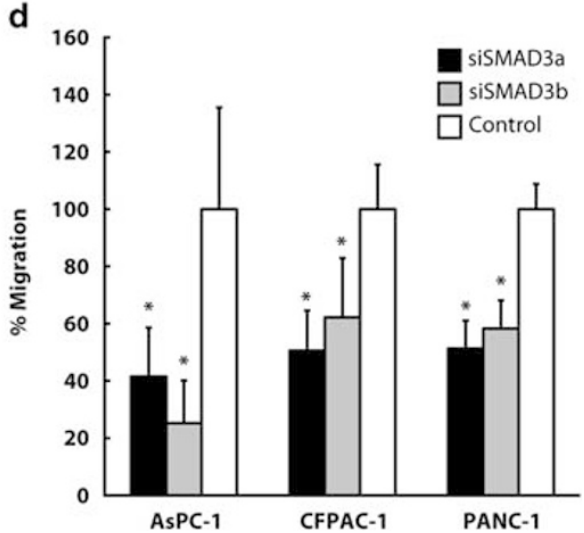

Figure 3 SMAD3 knockdown arrested the induction of EMT. (a) Western blots showed that two siRNA molecules targeting SMAD3 (siSMAD3a and siSMAD3b) were effective at reducing SMAD3 protein levels in AsPC-1 cells at 2 and 3 days after siRNA transfection. (b) AsPC-1, CFPAC-1, and PANC-1 cells were transfected with siSMAD3a or control siRNA and then treated with $5 \mathrm{ng} / \mathrm{ml} \mathrm{TGF-} \beta$ for $24 \mathrm{~h}$. Expression levels of SMAD3, E-cadherin (CDH1), and vimentin (VIM) in these cells were examined by quantitative RT-PCR. The expression levels were normalized to those of GAPDH in each cell line. The relative expression level shows the ratio of mRNA levels in siSMAD3a transfectants compared with the control cells without TGF- $\beta$ treatment (white bars). (c) SMAD3 knockdown resulted in morphological changes in AsPC-1, CFPAC-1, and PANC-1 cells. Scale bar $=100 \mu \mathrm{m}$. (d) Transwell migration assays showed a reduced number of migrated transfectants after SMAD3 knockdown compared with control cells. Error bars indicate the s.d. of the ratio of migrated cells/field (mean number of migrated cells in each control $=100 \%$ ) of experiments carried out in triplicate. Asterisks indicate a $P$-value of $<0.05$ compared with controls.

(Table 2). SMAD3 and solitary cell infiltration were identified as independent prognostic factors by multivariate analysis. Kaplan-Meier analyses showed that SMAD3 ${ }^{\text {high }}$ was associated with shorter overall survival (Figure 4a) and also with early recurrence (Figure 4c). Moreover, patients with both SMAD3 ${ }^{\text {high }}$ and SCI ${ }^{\text {high }}$ had a less favorable prognosis than others (Figures $4 \mathrm{~b}$ and $\mathrm{d}$ ). On the other hand, SMAD4 status was not associated with poor prognosis in this study (Supplementary Figure 7), whereas SMAD4 positivity correlated with EMT-like features in SMAD3 ${ }^{\text {high }}$ cases (Supplementary Table 3).

\section{DISCUSSION}

TGF- $\beta$ signaling is known to play a dual role in the regulation of proliferative and invasive properties of cancer. ${ }^{19,20}$ TGF- $\beta$ can induce EMT in PDAC cells, ${ }^{21,22}$ and overexpression of TGF- $\beta$ in PDAC correlates with decreased survival. ${ }^{9}$ In many gastrointestinal cancers, responses to TGF- $\beta$ are decreased by mutations or loss of expression of TGF- $\beta$ receptors and SMADs. ${ }^{23}$ Inactivation of SMAD4 through genetic aberrations occurs frequently in pancreatic and colorectal cancers. Although SMAD4 mutations are rare in gastric cancer, SMAD4 inactivation at the protein level is involved in 
Table 2 Univariate and multivariate analyses of EMT-like features associated with overall survival

\begin{tabular}{|c|c|c|c|c|}
\hline \multirow[b]{2}{*}{ EMT-like features } & \multicolumn{2}{|c|}{ Univariate } & \multicolumn{2}{|c|}{ Multivariate } \\
\hline & $\mathrm{HR}(95 \% \mathrm{Cl})$ & $P$-value & HR $(95 \% \mathrm{Cl})$ & $P$-value \\
\hline \multicolumn{5}{|l|}{ SMAD3 } \\
\hline Low vs high & $2.829(1.628-4.918)$ & $<0.001$ & $1.843(1.006-3.377)$ & 0.048 \\
\hline Reduced vs preserved & $0.529(0.304-0.922)$ & 0.025 & $0.875(0.475-1.612)$ & 0.875 \\
\hline \multicolumn{5}{|l|}{ Vimentin } \\
\hline Not expressed vs expressed & $2.833(1.637-4.926)$ & $<0.001$ & $1.795(0.971-3.317)$ & 0.062 \\
\hline
\end{tabular}

Abbreviations: $\mathrm{Cl}$, confidence interval; $\mathrm{HR}$, hazard ratio.
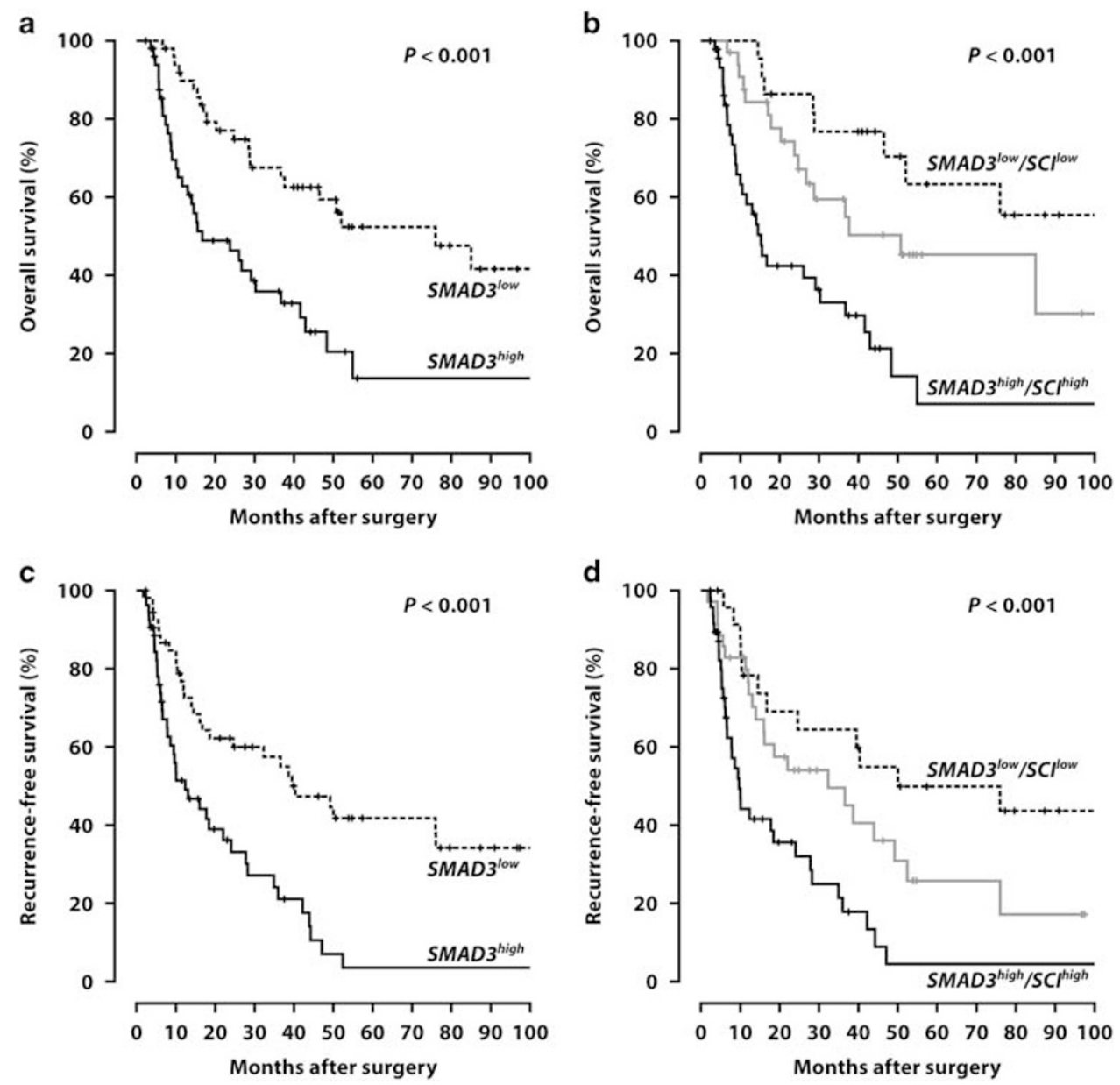

Figure 4 Kaplan-Meier survival analyses. Overall survival $(\mathbf{a}, \mathbf{b})$ and recurrence-free survival $(\mathbf{c}, \mathbf{d})$ were compared among patients with SMAD3 ${ }^{\text {low }}$ and $\operatorname{SMAD}^{\text {high }}(\mathbf{a}, \mathbf{c})$, or SMAD3 $3^{\text {low }} / \mathrm{SCl}^{\text {low }}, \mathrm{SMAD}^{\text {high }} / \mathrm{SCl}{ }^{\text {high }}$, and others (gray lines) $(\mathbf{b}, \mathbf{d})$. The $P$-value of the log-rank test for each analysis is indicated. 
gastric cancer progression. ${ }^{24}$ In our study, 67/113 cases (59\%) were SMAD4 negative. As immunohistochemical labeling for SMAD4 mirrors genetic status, ${ }^{25}$ SMAD4 was most likely inactivated in the SMAD4-negative cases.

TGF- $\beta$-induced EMT has been thought of as a SMAD4dependent process. Here, we show that EMT-like features, such as reduced E-cadherin, elevated vimentin, and increased cell motility, were not induced in SMAD4-inactivated AsPC-1 and CFPAC- 1 cells by TGF- $\beta$ treatment, whereas TGF- $\beta$ treatment significantly induced EMT-like features in SMAD4positive PANC-1 cells. These findings are in agreement with a previous report from Ellenrieder et al. ${ }^{21}$ However, some researchers have reported that TGF- $\beta$-induced EMT can also occur in a SMAD4-independent manner. ${ }^{26,27}$ TGF- $\beta$ can induce the activation of the mitogen-activated protein kinase (MAPK) signaling pathway that is involved in EMT induction independently of the canonical TGF- $\beta$ signaling pathway. ${ }^{26}$ Levy et al ${ }^{27}$ also showed that TGF- $\beta$-induced EMT is independent of SMAD4 and suggested that SMAD4-independent noncanonical TGF- $\beta$ signaling pathway or other pathways may be involved in EMT induction. Thus, the mechanism involved in TGF- $\beta$-induced EMT is still controversial.

Interestingly, we observed that SMAD3 knockdown in the cells without TGF- $\beta$ treatment also resulted in suppression of EMT-like features. Therefore, to induce EMT, SMAD3 may be involved in another pathway in addition to the canonical TGF- $\beta$ signaling pathway. Zhao et al ${ }^{28}$ reported that signal transducer and activator of transcription 3 (STAT3) is activated in SMAD4-inactivated PDACs and suggested that loss of SMAD4, leading to aberrant activation of STAT3, contributes to the switch of TGF- $\beta$ from a tumor-suppressive to a tumor-promoting pathway. As SMAD3 upregulation correlated with EMT-like features in PDACs regardless of SMAD4 status, it remains unclear whether SMAD3 cooperates with STAT3 to induce EMT in SMAD4inactivated cases.

Here, we examined gene expression profiles of PDACs on the basis of solitary cell infiltration to identify other EMTrelated genes that may increase the malignant potential of PDAC. These gene expression profiles revealed that enhanced expression of the SMAD3 gene was associated with EMT in PDAC. Immunohistochemical analysis confirmed that SMAD3 expression was mostly undetectable in nonneoplastic epithelia of the pancreas, but was readily apparent in the nuclei of tumor cells, despite the heterogeneous positivity observed among our cases. SMAD3 expression levels in PDAC cells were commonly high compared with other gastrointestinal (colon and stomach) cancer cells. SMAD3 overexpression correlates with Gleason score and expression of proliferating cell nuclear antigen in prostate cancer. ${ }^{29}$ Here, we found that high SMAD3 immunopositivity correlated with larger tumor size, major vessel involvement, higher tumor grade, and lymph node metastasis, all of which are characteristics associated with a poor prognosis in PDAC patients. ${ }^{2-4}$ Moreover, SMAD3 ${ }^{\text {high }}$ patients had a shorter survival time after surgery than $\mathrm{SMAD} 3^{\text {low }}$ patients. These results suggest that enhanced SMAD3 expression may facilitate the malignant progression of PDAC.

EMT-like features in PDAC have been implicated as biomarkers for poor prognosis. ${ }^{5,13,30}$ Here, we showed that high SMAD3 immunopositivity was associated with vascular invasion and lymph node metastasis as well as EMT-like features, suggesting that SMAD3-mediated EMT may promote invasion and metastasis, thereby linking it to poor prognosis. Besides SMAD3 ${ }^{\text {high }}$, we also identified $\mathrm{SCI}^{\text {high }}$ as another independent predictor of poor prognosis. The group of patients with both SMAD3 ${ }^{\text {high }}$ and SCI ${ }^{\text {high }}$ had the least favorable prognosis compared with other groups. In vitro analysis revealed that SMAD3 knockdown resulted in reduced cell motility, suggesting that SMAD3 may be involved in cell migration. In 113 cases of PDAC, SMAD $3^{\text {high }}$ significantly correlated with $\mathrm{SCI}^{\text {high }}$, and both were found to be independent factors for poor prognosis. Although it is clear that SMAD3 is involved in the malignancy process, most likely by inducing EMT, it is possible that the malignancy process is mediated by SMAD3 via another mechanism as well. EMT has been shown to result in cancer cells with stem cell-like characteristics that have a propensity to invade surrounding tissue and display resistance to certain therapies. ${ }^{31}$ Sun et al ${ }^{32}$ showed that NANOG promotes liver cancer cell invasion by inducing EMT through NODAL/SMAD3 signaling pathway. In human embryonic stem cells, SMAD2 and SMAD3 are involved in the transcriptional regulation of OCT4 and $N A N O G^{33}$ and control of the balance between self-renewal and differentiation. ${ }^{34}$ These findings may provide clues to clarify a possible mechanism underlying the malignant process of PDACs mediated by SMAD3.

In conclusion, enhanced expression of SMAD3 in PDAC correlated with malignant characteristics including EMT-like features, lymph node metastasis, and poor prognosis. SMAD3 seems to play an important role in malignant progression through EMT induction in PDAC, and may provide a potential biomarker for poor prognosis of PDAC. Supplementary Information accompanies the paper on the Laboratory
Investigation website (http://www.laboratoryinvestigation.org)

\section{ACKNOWLEDGMENTS}

This study was supported by grants from The New Energy and Industrial Technology Development Organization (Project no. P10003) and from The Ministry of Health, Labour and Welfare of Japan (Grant-in-aid of The Third Term Comprehensive 10-Year Strategy for Cancer Control).

\section{DISCLOSURE/CONFLICT OF INTEREST}

The authors declare no conflict of interest.

1. Jemal A, Bray F, Center MM, et al. Global cancer statistics. CA Cancer J Clin 2011;61:69-90. 
2. Trede M, Schwall G, Saeger HD. Survival after pancreatoduodenectomy. 118 consecutive resections without an operative mortality. Ann Surg 1990;211:447-458.

3. Yeo CJ, Cameron JL, Lillemoe KD, et al. Pancreaticoduodenectomy for cancer of the head of the pancreas. 201 patients. Ann Surg 1995;221:721-731.

4. Lüttges J, Schemm S, Vogel I, et al. The grade of pancreatic ductal carcinoma is an independent prognostic factor and is superior to the immunohistochemical assessment of proliferation. J Pathol 2000;191: 154-161.

5. Masugi Y, Yamazaki K, Hibi T, et al. Solitary cell infiltration is a novel indicator of poor prognosis and epithelial-mesenchymal transition in pancreatic cancer. Hum Pathol 2010;41:1061-1068.

6. Thiery JP. Epithelial-mesenchymal transitions in tumor progression. Nat Rev Cancer 2002;2:442-454.

7. Thiery JP, Acloque $\mathrm{H}$, Huang RYJ, et al. Epithelial-mesenchymal transitions in development and disease. Cell 2009;139:871-890.

8. Thiery JP, Sleeman JP. Complex networks orchestrate epithelialmesenchymal transitions. Nat Rev Mol Cell Biol 2006;7:131-142.

9. Friess $\mathrm{H}$, Yamanaka $\mathrm{Y}$, Büchler $\mathrm{M}$, et al. Enhanced expression of transforming growth factor beta isoforms in pancreatic cancer correlates with decreased survival. Gastroenterology 1993;105:1846-1856.

10. Tascilar M, Skinner HG, Rosty C, et al. The SMAD4 protein and prognosis of pancreatic ductal adenocarcinoma. Clin Cancer Res 2001;7:4115-4121.

11. Fink SP, Mikkola D, Willson JKV, et al. TGF-beta-induced nuclear localization of Smad2 and Smad3 in Smad4 null cancer cell lines. Oncogene 2003;22:1317-1323.

12. Blackford A, Serrano OK, Wolfgang $\mathrm{CL}$, et al. SMAD4 gene mutations are associated with poor prognosis in pancreatic cancer. Clin Cancer Res 2009:15:4674-4679.

13. Shimamura $T$, Sakamoto $M$, Ino $Y$, et al. Dysadherin overexpression in pancreatic ductal adenocarcinoma reflects tumor aggressiveness: relationship to e-cadherin expression. J Clin Oncol 2003;21:659-667.

14. Yamazaki K, Takamura M, Masugi Y, et al. Adenylate cyclase-associated protein 1 overexpressed in pancreatic cancers is involved in cancer cell motility. Lab Invest 2009;89:425-432.

15. Hruban $\mathrm{RH}$, Boffetta $\mathrm{P}$, Hiraoka $\mathrm{N}$, et al. Tumors of the pancreas. In: Bosman FT, Carneiro F, Hruban RH, et al. (eds). World Health Organization Classification of Tumors of the Digestive System, Lyon, France, 2010, pp 279-337.

16. Uchida $\mathrm{H}$, Yamazaki $\mathrm{K}$, Fukuma $\mathrm{M}$, et al. Overexpression of leucine-rich repeat-containing $\mathrm{G}$ protein-coupled receptor 5 in colorectal cancer. Cancer Sci 2010;101:1731-1737.

17. Schutte $M$, Hruban $R H$, Hedrick $L$, et al. DPC4 gene in various tumor types. Cancer Res 1996;56:2527-2530.

18. Moore PS, Sipos B, Orlandini S, et al. Genetic profile of 22 pancreatic carcinoma cell lines. Analysis of K-ras, p53, p16 and DPC4/Smad4. Virchows Arch 2001;439:798-802.

19. Welch DR, Fabra A, Nakajima M. Transforming growth factor beta stimulates mammary adenocarcinoma cell invasion and metastatic potential. Proc Natl Acad Sci USA 1990;87:7678-7682.
20. Markowitz SD, Roberts AB. Tumor suppressor activity of the TGFbeta pathway in human cancers. Cytokine Growth Factor Rev 1996;7: 93-102.

21. Ellenrieder V, Hendler SF, Boeck W, et al. Transforming growth factor beta1 treatment leads to an epithelial-mesenchymal transdifferentiation of pancreatic cancer cells requiring extracellular signal-regulated kinase 2 activation. Cancer Res 2001;61:4222-4228.

22. Jungert K, Buck A, Buchholz M, et al. Smad-Sp1 complexes mediate TGFbeta-induced early transcription of oncogenic Smad7 in pancreatic cancer cells. Carcinogenesis 2006;27:2392-23401.

23. Achyut BR, Yang L. Transforming growth factor- $\beta$ in the gastrointestinal and hepatic tumor microenvironment. Gastroenterology 2011;141:1167-1178.

24. Wang L, Kim S, Lee JH, et al. Inactivation of SMAD4 tumor suppressor gene during gastric carcinoma progression. Clin Cancer Res 2007;13 102-110.

25. Wilentz RE, Su GH, Dai JL, et al. Immunohistochemical labeling for dpc4 mirrors genetic status in pancreatic adenocarcinomas: a new marker of DPC4 inactivation. Am J Pathol 2000;156:37-43.

26. Giehl K, Imamichi Y, Menke A. Smad4-independent TGF-beta signaling in tumor cell migration. Cells Tissues Organs 2007;185: 123-130.

27. Levy L, Hill CS. Smad4 dependency defines two classes of transforming growth factor $\beta$ (TGF- $\beta$ ) target genes and distinguishes TGF- $\beta$-induced epithelial-mesenchymal transition from its antiproliferative and migratory responses. Mol Cell Biol 2005;25:8108-8125.

28. Zhao S, Venkatasubbarao K, Lazor JW, et al. Inhibition of STAT3 Tyr705 phosphorylation by Smad4 suppresses transforming growth factor $\beta$-mediated invasion and metastasis in pancreatic cancer cells. Cancer Res 2008;68:4221-4228.

29. Lu S, Lee J, Revelo $M$, et al. Smad3 is overexpressed in advanced human prostate cancer and necessary for progressive growth of prostate cancer cells in nude mice. Clin Cancer Res 2007:13: 5692-5702.

30. Javle MM, Gibbs JF, Iwata KK, et al. Epithelial-mesenchymal transition (EMT) and activated extracellular signal-regulated kinase (p-Erk) in surgically resected pancreatic cancer. Ann Surg Oncol 2007;14: 3527-3533.

31. Polyak K, Weinberg RA. Transitions between epithelial and mesenchymal states: acquisition of malignant and stem cell traits. Nat Rev Cancer 2009;9:265-273.

32. Sun $C$, Sun $L$, Jiang $K$, et al. NANOG promotes liver cancer cell invasion by inducing epithelial-mesenchymal transition through NODAL/SMAD3 signaling pathway. Int J Biochem Cell Biol 2013;45:1099-1108.

33. Brown S, Teo A, Pauklin S, et al. Activin/Nodal signaling controls divergent transcriptional networks in human embryonic stem cells and in endoderm progenitors. Stem Cells 2011;29:1176-1185.

34. Singh AM, Reynolds D, Cliff $T$, et al. Signaling network crosstalk in human pluripotent cells: a Smad2/3-regulated switch that controls the balance between self-renewal and differentiation. Cell Stem Cell 2012;10:312-326. 